\title{
A Whole-Body Approach to Compare Different Lifting Styles
}

\section{Mohammad Abdoli-Eramaki ${ }^{*}$, Sanaz Agha ${ }^{2}$ and Hamed Pardehshenas ${ }^{3}$}

${ }^{1}$ Associate Professor, School of Occupational and Public Health, Ryerson University, Toronto, Canada

${ }^{2}$ Rehabilitation Science, Research Assistant, Rehabilitation Science Institute, University of Toronto, Toronto, ON, Canada

${ }^{3}$ Research Assistant, Occupational Biomechanics, Ergonomics and Injury Prevention Lab, Ryerson University, Toronto, Canada

*Corresponding Author: Mohammad Abdoli-Eramaki, Associate Professor, School of Occupational and Public Health, Ryerson University, Toronto, Canada.

Received: September 11, 2019; Published: October 11, 2019

DOI: $10.31080 /$ ASOR.2019.02.0109

\begin{abstract}
Objective: To challenge six most common lift styles to capture the concept of minimal task demand using EMG and motion capture system. It seems feasible to expect that a change in lifting style should work and result concurrently in most adaptable and safest practice of lifting tasks.

Methodology: The external dynamic moments and surface muscle activities were utilized as indicators for comparison among six lifting styles: a) modified full-squat, b) BLT, c) sumo, d) semi-squat, e) straddle, and f) stoop lift. A repeated-measures design was used to test the main effect of lift style on net dynamic external moments and muscles' activation level.

Results: Lift style had a significant influence on the normalized resultant moment for the low back, hip, knee, and ankle joints; but, no effect on the shoulder. No significant difference was found in low back moment among BLT, sumo, and squat $(\mathrm{p}<1.000)$. The semisquat created the lowest knee moment ( $\mathrm{p}<0.001$ ), by at least $34 \%$ lower than BLT. Also, the semi-squat caused the highest erector spinae level of activity by at least $16 \%$ higher than BLT $(\mathrm{p}<0.007)$. The maximal difference was observed for the Sumo by $20 \%$ difference $(\mathrm{p}<0.007)$.

Conclusion: It seems that for a single lifting task, semi-squat is the reasonable compromise among all techniques, by keeping the resultant joint moments at the lowest range. However, for repetitive lifting tasks; where local and whole body fatigue can lead to MSK injuries, sumo appears to be the best compromise.

Application: The results of this study may help better understand why workers fail to follow training instructions, which can facilitate the development of effective training programs for the trainers and workers accordingly. The outcome of this study will apply to workplace training programs and future research investigations into the effectiveness of lift training.

Specific: It seems feasible to expect that a change in training content of manual material handling (lifting technique) should work and result concurrently in most adaptable and safest practice of lifting tasks. This study is a first attempt to examine the concept of minimal energy expenditure during different lifting techniques.
\end{abstract}

Keywords: Manual Material Handling; Lifting Style; Biomechanics; Musculoskeletal Injuries

\section{Introduction}

Manual material handling can set in motion a series of events leading to neuromusculoskeletal (NMSK) disorders such as low back pain [1-7]. Training is an essential element of any workplace injury prevention, health promotion, or return-to-work programs. Since musculoskeletal disorders are prevalent among manual 
handling workers, safe lifting technique is necessary [8]. Despite many research studies and training programs on the prevention of low back injury, it is still an ongoing challenge in the workplace, leading all other body parts with $17 \%$ of injuries [9]. Moreover, despite the need for training, the evidence suggests that lift training is ineffective at transferring skills as workers tend to go back to their past practice [10-14]. The issue could be partly explained by training methods $[15,16]$, but the training content might need to be evaluated as well. The content of lift training, specifically the lift style, has been controversial because of inconclusive evidence on the best lift style [17]. Some studies have explored alternative lift styles such as semi-squat [10], modified full-squat [18], straddle [19,20] and weightlifters' technique [21]. The lifting scenarios represent a set of relevant lifting techniques, but no one can infer that these lifting techniques can be easily applied in all workplace settings where repetitive lifting might result in different motor control strategies. According to biomechanical studies, the semi-squat style was introduced as the safest and most effective basis for lifting training $[10,17]$. However, the result is challenged by physiological results indicating that stoop lifting has the lower energy expenditure, cardio-respiratory demands, and perceived exertion [22].

According to one of the latest motor control theories, individuals adapt their walking pattern in response to changing conditions to lower their energy consumption [23,24]. In these gait studies, they intentionally changed the walking conditions and found that walkers quickly adapted their technique to minimize energy demand. This knowledge can help to determine a posture during the lifting task that optimally requires the minimum rate of energy and balanced moments on the joints.

In this study, several lifting techniques were compared using the concepts of balancing the moments across the joints involved in the lifting task, and a surrogate measure of energy demand namely summated muscle activity.

The study aimed to challenge six most common lift styles to capture the concept of minimal task demand using EMG and motion capture system. It seems feasible to expect that a change in lifting style (training content as a part of behavioral control) should work and result concurrently in most adaptable and safest practice of lifting tasks. The results of this study will help better understand why workers fail to follow training instructions, which can facili- tate the development of effective training programs for the trainers and workers accordingly. The outcome of this study will apply to workplace training programs and future research investigations into the effectiveness of lift training.

\section{Methods}

\section{Experimental design}

The external dynamic moments and surface muscle activities were utilized as indicators for comparison among six lifting styles in this study: a) modified full-squat, b) BLT, c) sumo, d) semi-squat, e) straddle, and f) stoop lift from floor to table-height (Figure 1). These selected posture are the most common methods that are studied by other researchers or trained in workplaces. A repeatedmeasures design was used to test the main effect of lift style on net dynamic external moments (L4/L5, shoulder, hip, knee, and ankle), and on muscle activity (erector spinae (ES), latissimus dorsi (LD), internal oblique (IO), lateral gastrocnemius (LG), gluteus medius (GM), biceps femoris (BF), rectus femoris (RF), and adductor longus (AL)). Lift styles were tested in a randomized order.

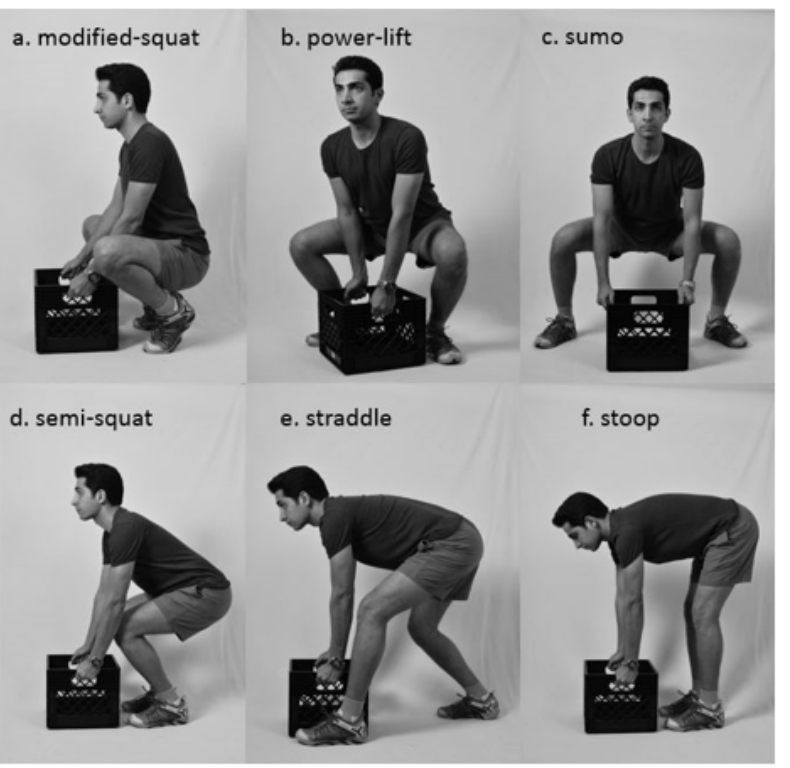

Figure 1: Six lifting styles: a) modified full-squat, b) balanced lifting technique, c) sumo, d) semi-squat, e) straddle, and f) stoop.

\section{Participants}

Participants were recruited from the university population; anyone with a history of musculoskeletal pain or injury within the 
previous six months or health concerns precluding participation in strenuous activity were excluded, based on responses to the Physical Activity Readiness Questionnaire [25]. A total of 19 males (10) and females (9) with the age $=24.4$ (SD 6.1) years, height $=168.4$ $($ SD 12.0$) \mathrm{cm}$, and body mass $=65.5$ (SD 14.7) $\mathrm{kg}$ participated after providing written, informed consent. The methodology was approved by the Ryerson University Research Ethics Board.

\section{Set up and procedures}

Starting in upright standing posture, participants reached down to pick up a weighted crate (13" square x 11" height) from the floor to elbow height, and then lowered the crate back to the floor, to its original position. The load lifted was $20 \%$ of the participant's body mass as a weight that almost $75 \%$ of males and females can lift. Each of the six lift styles was repeated three times; between trials and repetitions, a 2-minute break was provided to avoid early onset of muscle fatigue. Lift pace was standardized using a metronome set at a comfortable pace of 38-40 beats per minute. The pace was established during the training session explained below.

The six lift styles were taught and practiced before testing at a separate training session for about 15 minutes (Figure 1) for an average of about 10 lift for each style to establish a rhythm in their lifting. Participants who had a difficulty with a style e.g. straddle were asked to practice to a point that they could comfortably complete the lift. For all lift styles, the instruction was to "stand upright behind the crate, then reach the load, raise the crate to elbow height, put it back in the original position, and return to the upright position." As demonstrated in Figure 1, in "modified full-squat" the feet and knees were instructed to be rotated outwards by approximately $45^{\circ}$. The participant was cued to "maintain a lumbar lordosis throughout all the lifting activities, as much as possible" (Figure 1.a to 1.f). In "balanced lifting technique" or BLT the feet were positioned almost at a right angle, and the load is approached from one of its corners (Figure 1.b). Cueing for BLT was "keep your chest up and your back neutral." In sumo squat, the feet were positioned further apart than shoulder width, and the toes are slightly turned outward (Figure 1.c). Cueing for sumo squat was "keep your chest up and your back neutral for the duration of the movement, and knees aligned with the ankles. The instruction for semisquat was "lower yourself into a semi-squatting position by bending at hip and knee joints." The participant was cued to "keep your head upright and low back straight while maintaining the lumbar curve" (Figure 1.d). In stoop style, the participant was instructed to lean over, flex the hip joints and the back while keeping their legs straight (knees almost locked). The participant was also cued to "maintain the lumbar curve as much as possible" (figure 1.e). In straddle style, the instruction was to place one foot beside the crate and the other behind the crate [20] "while keeping the back straight" (figure 1.f).

\section{Instrumentation, sensor location, and data collection}

The 3-dimensional orientation of seven body segments (nondominant thigh, lower leg, foot, arm and forearm, pelvis and trunk) were continuously recorded at $120 \mathrm{~Hz}$ using the Fastrak ${ }^{\mathrm{TM}}$ electromagnetic tracking system (Polhemus, Colchester, VT, USA). Sensors were attached firmly to the skin using Kinesio Tape (C) (KT Health, American Fork, UT, USA). Sensors located on the extremities were positioned at the approximate midpoint of each segment. The sensor tracking pelvis orientation was positioned overlying the anterior superior iliac spine on the non-dominant side. Trunk orientation was tracked using two sensors: one sensor was positioned overlying the spinous process of seventh cervical vertebrae, and the second was overlying L4/L5. The electromagnetic source was positioned within 1 meter of all sensors with no metal within the active field.

Orientation was normalized to upright standing, using a standing reference position that was digitized before every repetition. For this, participants were instructed to 'stand upright, with arms at the body's sides with the palms facing inward.

Surface muscle activity was continuously recorded at $2048 \mathrm{~Hz}$ using a Bortec (Bortec Biomedical Ltd. Calgary, AB, CA) EMG system (Input Impedance $10 \mathrm{G} \Omega, \mathrm{CMRR}>115 \mathrm{~dB}$ ). Electrodes were positioned overlying eight muscles on the dominant side. Before placement, the location was marked, then the skin shaved, sanded, and then cleaned with an alcohol swab. Surface, pre-gelled disposable, bipolar EMG electrodes (Bortec Biomedical Ltd. Calgary, AB, CA) were used with $2 \mathrm{~cm}$ inter-electrode distance; these were secured with Kinesio Tape(C). Locations were chosen by previous anatomical investigations $[26,27]$ and Surface Electromyography for the Non-Invasive Assessment of Muscles [28]. The Biceps Femoris (BF) electrode was placed midway between the ischial tuberosity and the caput fibulae. For Leg Gastrocnemius (LG), the electrode was placed at a $1 / 3^{\text {rd }}$ distance of the line between the head of the fibula 
and the heel. The electrode for Latissimus Dorsi (LD) was placed over the muscle belly at the T12 level and on the line connecting the most superior point of the posterior axillary fold and the S2 spinous process. For Erector Spinae (ES), electrodes were placed on the line from the caudal tip of the posterior superior iliac spine (PSIS) to the interspace between L1 and L2 spinous process at the level of L5 spinous process (i.e., about $2-3 \mathrm{~cm}$ from the midline). For the Gluteus Medius (GM), the electrode was placed at $50 \%$ on the line from the crista iliaca to the greater trochanter of the femur. For Adductor Longus (AL), the attachment site was over the muscle belly, in the medial region of the thigh, in an oblique direction, four centimeters below the pubis [29]. The electrodes were placed at $50 \%$ on the line from the anterior superior iliac spine to the superior part of the patella to record RF muscle. For Internal Obliques (IO), electrodes were placed $2 \mathrm{~cm}$ medial and inferior to the anterior superior iliac spine and beneath the line joining both anterior superior iliac spine [30]. The ground reference electrode was placed overlying the patella.

Muscle activity was calibrated to a maximal isometric voluntary contraction (MVIC) using recommended procedures in SENIAM that was completed on the same day before testing. The average value of three repetitions lasting for 3-4 seconds was used for calibration. All the procedures were conducted by the same researcher for all participants to minimize inter-rater error. Both visual and verbal cueing was provided to reach maximal isometric efforts. A 2-minute rest was given between MVIC repetitions to avoid muscular fatigue.

\section{Data processing and calculations}

A custom program was developed in Labview 8.5 (National Instruments, Austin, TX, USA) to estimate the three-dimensional dynamic moments about the shoulders, L4/L5, hips, knees, and ankles joints as described by Hof., et al. [31] and Plamondon., et al. [32]. All Fastrak ${ }^{\mathrm{TM}}$ data were processed with a second-order lowpass Butterworth filter at $10 \mathrm{~Hz}$. This approach involved moving the inertial forces due to linear accelerations to the centre of masses of the segments. The linear velocities and linear accelerations of each segment were computed by numerical differentiation procedures using the central-difference method. Three dimensional moments due to segment rotations were found from each segment's angular rotation for the third part of the Hof equation (1992). Angular velocities were determined by the method proposed by Paul
(1981), where each segment was expressed in a global coordinate system using a matrix of direction cosines; angular velocities were then determined by the product of the derivative of this matrix. The calculated static and dynamic moments were summed together for each separate segment and for all of the segments with each other. Kinematic data were chopped from the erect standing posture to the beginning of the lift and normalized to $100 \%$ of the lifting cycle.

The linear acceleration of each segment and load was computed as the second derivative of displacement and then filtered using a second-order Butterworth filter with a cutoff frequency of 4 $\mathrm{Hz}$ [31]. From these curves, peak moments and root mean square (RMS) values were determined and processed for statistical analysis. The average of three repetitions at each time point for each lift style was used for comparison. All moments were normalized by bodyweight times height method to reduce variability among the moment results.

Raw EMG data for each trial was linear enveloped by rectifying and low-pass filtering using a $2^{\text {nd }}$ order Butterworth filter with a 2.5 $\mathrm{Hz}$ cut-off. The RMS was then calculated and normalized using the MVIC value. For each trial, the synchronized kinematic data were used to determine the percent time of the lifting cycle. These three trials per muscle were then averaged, and outcome variables were calculated.

\section{Statistical analysis}

The extracted findings for comparison were first tested for normal distribution using the Shapiro-Wilk statistic. A one-way repeated-measures analysis of variance (ANOVA) was used to test for the main effect from lift style. The sphericity of the data was tested using Mauchly's test. Where Sphericity could not be assumed, the Greenhouse-Geisser correction was used to adjust the degrees of freedom of the repeated measures ANOVA. Bonferroni pairwise comparison tests were used to examine differences between lift styles. Analyses were performed using version 22.0 of Statistical Package for the Social Sciences (SPSS) with a significance level of $\alpha$ $=0.05$ for all tests. Also, using the concept that humans adopt strategies that minimize energy consumption [24], all muscles were included to form a composite of total activity. Then, the coefficient of variation (CV) between all muscles' activation level was calculated for all techniques as a measure of uniformity (variability) of muscle activation pattern. 


\section{Results}

Results were tested for normal distribution using the ShapiroWilk in SPSS for Windows, release 22.0 (SPSS, Corporation, Chicago, IL, USA). A one-way repeated-measures analysis of variance (ANOVA) was used to test for the main effect for lift style. Statistical significance was accepted when $\mathrm{p}<.05$. Bonferroni pairwise comparison tests were used to examine differences between lift styles. Also, the coefficient of variation (CV) between all muscles' activation level was calculated for all techniques as a measure of uniformity (variability) of muscle activation pattern.

\section{External moments}

Lift style had a significant influence on the normalized resultant moment $(\mathrm{NRM})$ for the low back $[\mathrm{F}(5,60)=27.31, \mathrm{p}<0.0001]$, hip $[F(5,60)=27.71, p<0.0001]$, knee $[F(2.59,31.17)=11.34, p<$ $0.0001]$, and ankle $[F(5,60)=20.32, p<0.0001]$ joints; but, no effect on the shoulder NRM $[F(1.89,22.67)=0.987, p<0.38]$ (Table 1).

\begin{tabular}{|l|c|c|c|c|}
\hline NRM & df $_{\text {lift style }}$ & df $_{\text {Error (lift style) }}$ & F-statistics & P-value \\
\hline Back & 5 & 60 & 27.31 & $<0.0001$ \\
\hline Knee & 2.598 & 31.175 & 11.34 & $<0.0001$ \\
\hline Hip & 5 & 60 & 27.71 & $<0.0001$ \\
\hline Ankle & 5 & 60 & 20.32 & $<0.0001$ \\
\hline Shoulder & 1.89 & 22.678 & 0.987 & 0.38 \\
\hline
\end{tabular}

Table 1: A summary of the ANOVA results with F-statistics and corresponding $\mathrm{p}$-values for the main effect of lift style on normalized resultant moments (NRM).

Post hoc tests using the Bonferroni correction revealed that the modified full-squat causes lowest low back NRM ( $\mathrm{p}<0.003)$, by at least an average of $0.046 \mathrm{Nm} / \mathrm{H}^{*} \mathrm{~W}(34 \%)$ ( $\left.<<0.001\right)$, lower than sumo (figure 2.). On the other hand, stoop and straddle increased Low back NRM by at least an average of $0.047 \mathrm{Nm} / \mathrm{H}^{*} \mathrm{~W}$ $(24 \%)$ and $0.061(31 \%)$ more than BLT; respectively ( $p<0.012)$. No significant difference was found in low back NRM among BLT, sumo and squat $(\mathrm{p}<1.000)$. Post hoc tests demonstrated that the semi-squat lift style created the lowest knee NRM $(p<0.001)$, by at least an average of $0.113 \mathrm{Nm} / \mathrm{H}^{*} \mathrm{~W}(34 \%)$ lower than BLT (Figure 2). However, sumo created the highest ankle NRM, which was significantly higher than the modified full-squat (21\%), stoop (35\%) and $(46 \%)$ semi-squat ( $<<0.001$ ), but not significantly different in comparison to BLT and straddle $(\mathrm{p}<1.000)$. Post hoc tests showed in comparison to straddle and stoop which created the highest hip NRM (by at least an average of $0.053 \mathrm{Nm} / \mathrm{H}^{*} \mathrm{~W}(16 \%)$ higher than
BLT $(p<0.006)$ ), the modified full-squat caused the lowest hip NRM within all lifting styles (by at least an average of $0.053 \mathrm{Nm} / \mathrm{H}^{*} \mathrm{~W}$ $(16 \%)$ higher than BLT) $(\mathrm{p}<0.005)$. It could mean that there was no difference between semi-squat, BLT, and sumo.

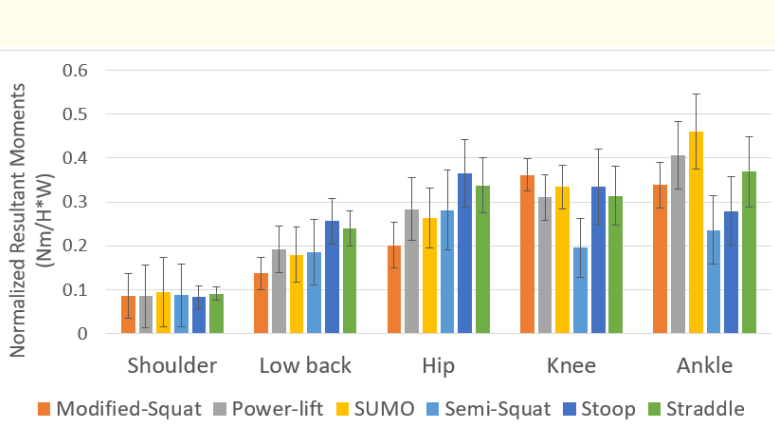

Figure 2: Main effect of lifting technique on normalized resultant moments in different joints. $(\mathrm{Nm}=$ newton meter, $\mathrm{H}=$ height, $\mathrm{w}=$ weight $)$.

Main effect of lifting technique on myoelectric activity of muscles (RMS values)

A summary of EMG results from the ANOVA with F-statistics, and corresponding p-values are shown in table 2.

\begin{tabular}{|l|c|c|c|c|}
\hline $\begin{array}{l}\text { Myoelectric } \\
\text { activity }\end{array}$ & $\mathbf{d f}_{\text {lift style }}$ & df $_{\text {Error (lift style) }}$ & F-statistics & P-value \\
\hline $\begin{array}{l}\text { Biceps Femo- } \\
\text { ris }\end{array}$ & 1.94 & 33.14 & 0.31 & 0.72 \\
\hline $\begin{array}{l}\text { Gastrocne- } \\
\text { mius }\end{array}$ & 2.35 & 40.01 & 1.84 & 0.16 \\
\hline $\begin{array}{l}\text { Latissimus } \\
\text { Dorsi }\end{array}$ & 1.54 & 26.17 & 1.41 & 0.25 \\
\hline $\begin{array}{l}\text { Lumbar Erec- } \\
\text { tor Spinae }\end{array}$ & 3.75 & 63.78 & 2.59 & 0.03 \\
\hline $\begin{array}{l}\text { Gluteus } \\
\text { Medius }\end{array}$ & 2.35 & 40.06 & 0.91 & 0.42 \\
\hline $\begin{array}{l}\text { Adductor } \\
\text { longus }\end{array}$ & 3.22 & 54.88 & 3.47 & 0.00 \\
\hline $\begin{array}{l}\text { Rectus Femo- } \\
\text { ris }\end{array}$ & 2.50 & 40.14 & 8.07 & 0.00 \\
\hline $\begin{array}{l}\text { Internal } \\
\text { Oblique }\end{array}$ & 3.05 & 48.88 & 1.38 & 0.25 \\
\hline
\end{tabular}

Table 2: A summary of EMG results from the ANOVA with Fstatistics and corresponding p-values. 
Post hoc tests using the Bonferroni correction revealed that among all techniques, stoop caused the substantially the lowest rectus femoris activity by at least 14.62 mean difference (370\%) lower than sumo. It also showed that the stoop and semi-squat caused the lowest and highest adductor longus activity $(\mathrm{p}<0.001)$, respectively (Figure 3 ).

Also, the semi-squat technique caused the highest erector spinae level of activity by at least 3.24 mean difference (16\%) in comparison with BLT $(\mathrm{p}<0.007)$. The maximal difference was observed for the Sumo technique by $20 \%$ difference ( $\mathrm{p}<0.007$ ). Sumo style resulted in less rectus femoris and adductor longus activities comparing to Semi-Squat, but not statistically significant. There was no significant effect of the lifting condition among other muscles.

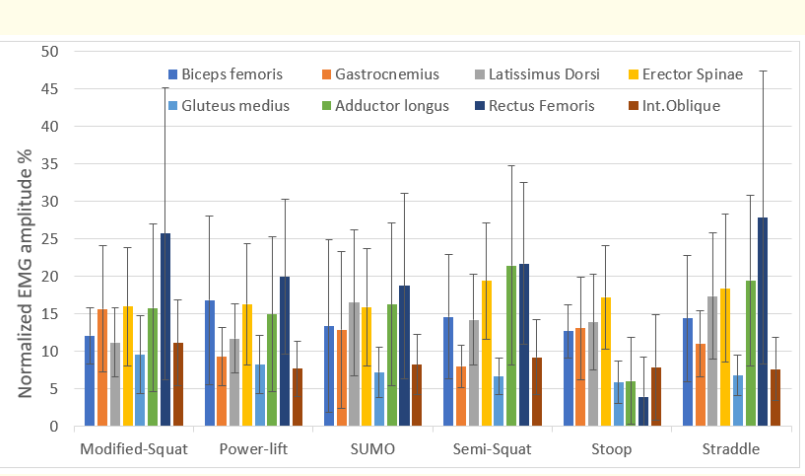

Figure 3: Mean (SD) of the normalized RMS amplitudes of muscles during six different lift styles.

\section{Discussion}

It has been shown that neuromuscular programming quickly evolves strategies to minimize energy costs during gait, even when the natural pattern must be changed to minimize energy [24]. When applied to lifting tasks, this theory would explain why workers' natural pattern might change from squat-like to stoop-like during prolonged, repetitive work because there is less energy demand. This study is a first attempt to examine this concept during different lifting techniques using joint moments and EMG findings.

The results from joint moments showed that the modified full-squat created the lowest hip and back moments while causing the highest knee moments. Obviously, stoop and straddle techniques created the highest back and hip moments, respectively.
Semi-squat showed the lowest knee and ankle moments which altogether with other moments, it seems to be the most optimal technique. It also indicates that when semi-squat is appropriately conducted, it might reduce the risk for the knees, ankles, low back, and hips joints by sharing the applied load at a moderate level. In other words, in comparison to extreme postures such as stoop and modified full-squat, the semi-squat provides a balanced posture that uniformly distributes the load on each joint accordingly. Then, semi-squat might be initiated first in workers with lower extremities injuries during gradual return to work.

In light of observations above, which are consistent with the proponents of semi-squat technique, it is arguable that, despite all training programs, workers shift from semi-squat to stoop especially following prolonged, repetitive lifting activities. One possible explanation is the fact that the human body always tends to move towards minimum energy expenditure [23,24]. Based upon the myoelectric activity of muscles measured in this study, we observed the lowest summated EMG activity during stoop technique, indicating the lowest muscular contribution (active elements) during this lifting task. The stoop was followed by sumo, BLT, and semi-squat, modified full-squat, and straddle; respectively. Then, it is reasonable from the energy consumption perspective that workers would tend toward the least energy-demanding strategy (i.e., stoop) despite training to the contrary, especially when workers are fatigued or have not identified any alternatives. Several good quality studies agree that stoop lifting resulted in the least total energy expenditure, heart rate and ventilation, $20-30 \%$ less than full-squat and 10-15\% less than semi-squat. It suggests workers using this technique are less likely to suffer injuries associated with whole-body fatigue despite its adverse effect on back moments. It is also suggested by Burgess-Limerick., et al. [33] that "a stooped posture has the advantage of lowering the center of gravity of the upper body less than a semi-squat posture and thus less work is done in lifting the upper body during each lift". After stoop, sumo was the most efficient style regarding the summated muscular activity. Also, considering overall muscular activation pattern, sumo style showed the most uniform pattern of activity by having the lowest coefficient of variation within all muscles measured in this study. In other words, it seems that sumo engages all body parts by incorporating all muscles at an optimal level during the lifting task, similar to what was observed in terms of moment's sharing during semisquat. Additionally, comparing to semi-squat, results showed sumo 
lifting reduced erector spinae muscle activity, around 20\%. Based on the results, it seems that the movement pattern during sumo style can engage both active and passive properties of the muscles, which reduces the muscular effort required to perform the task. It suggests that the sumo appears to be most optimal and efficient style among all techniques regarding muscular effort and should be studied further. On the other hand, the sumo style did not create significant different low back and hip moments in comparison to semi-squat technique thus indicating that these moments are in an acceptable range.

\section{Conclusion}

It seems that for a single lifting task, semi-squat is the reasonable compromise among all techniques, by keeping the resultant joint moments at the lowest range. However, for repetitive lifting tasks; where local and whole body fatigue can lead to MSK injuries, sumo appears to be the best compromise. Perhaps workers can be trained to alternate between semi-squat and sumo styles as they fatigue rather than resorting to the squat lifting style. Also, workers with underlying musculoskeletal injuries can be trained to alternate between different lifting techniques according to the nature and recovery status of their injuries. These types of laboratory studies should be completed before training the workers on the job.

\section{Key points}

- The concept of workers self-selecting a lifting style that best conserve their energy is novel. This concept has been verified in human gait [24], and it is logical that the same principles could explain why many workers naturally select a stoop lifting style, regardless of training.

- $\quad$ Although there are limitations in this study (e.g., sample size, lifting experience, number of muscles and joints studied), it demonstrates that we must consider more than joint moments to understand the biomechanics of the lifting task and why many training programs are ineffective.

- $\quad$ Future studies should be devoted to training using the semi-squat and sumo styles as well as determining how these lifting styles are altered as workers fatigue.

\section{Bibliography}

1. Hoogendoorn WE., et al. "Flexion and rotation of the trunk and lifting at work are risk factors for low back pain: results of a prospective cohort study". Spine 25 (2000): 3087-3092.
2. Hoozemans MJ., et al. "Pushing and pulling in relation to musculoskeletal disorders: a review of risk factors". Ergonomics 41 (1998): 757-781.

3. Norman R., et al. "A comparison of peak vs cumulative physical work exposure risk factors for the reporting of low back pain in the automotive industry". Clinical Biomechanics 13 (1998): 561-573.

4. Widanarko B., et al. "Prevalence of musculoskeletal symptoms in relation to gender, age, and occupational/industrial group". International Journal of Industrial Ergonomics 41 (2011): 561572.

5. Coenen P., et al. "The effect of lifting during work on low back pain: a health impact assessment based on a meta-analysis". Occupational and Environmental Medicine (2014): 102346.

6. Coenen P., et al. "Cumulative mechanical low-back load at work is a determinant of low-back pain". Occupational and Environmental Medicine 71 (2014b): 332-337.

7. Antwi-Afari M., et al. "Biomechanical analysis of risk factors for work-related musculoskeletal disorders during repetitive lifting task in construction workers". Automation in Construction 83 (2017): 41-47.

8. Yeung SS., et al. "Prevalence of musculoskeletal symptoms in single and multiple body regions and effects of perceived risk of injury among manual handling workers". Spine 27 (2002): 2166-2172.

9. 2015 WSIB Statistical Report.

10. Burgess-Limerick R. "Squat, stoop, or something in between?". International Journal of Industrial Ergonomics 31 (2003): 143148.

11. Clemes SA., et al. "What constitutes effective manual handling training? A systematic review". Occupational Medicine 60 (2009): 101-107.

12. Kroemer K. "Personnel training for safer material handling". Ergonomics 35 (1992): 1119-1134.

13. Verbeek J., et al. "Manual material handling advice and assistive devices for preventing and treating back pain in workers: a Cochrane Systematic Review". Occupational and Environmental Medicine (2011): 100214. 
14. Hogan DA., et al. "The effect of manual handling training on achieving training transfer, employee's behaviour change and subsequent reduction of work-related musculoskeletal disorders: a systematic review". Ergonomics 57 (2014): 93-107.

15. Burke MJ., et al. "Relative effectiveness of worker safety and health training methods". American Journal of Public Health 96 (2006): 315-324.

16. van der Molen HF., et al. "Effectiveness of measures and implementation strategies in reducing physical work demands due to manual handling at work". Scandinavian Journal of Work Environment and Health (2005): 75-87.

17. Sedgwick A and Gormley. "Training for lifting; an unresolved ergonomic issue?”. Applied Ergonomics 29 (1998): 395-398.

18. Kingma I., et al. "Foot positioning instruction, initial vertical load position and lifting technique: effects on low back loading". Ergonomics 47 (2004): 1365-1385.

19. de Looze M.P., et al. "Does an asymmetric straddle-legged lifting movement reduce the low-back load?". Human Movement Science 17 (1998): 243-259.

20. Kingma I., et al. "Can low back loading during lifting be reduced by placing one leg beside the object to be lifted?". Physical Therapy 86 (2006): 1091.

21. Kingma I., et al. "How to lift a box that is too large to fit between the knees". Ergonomics 53 (2010): 1228-1238.

22. Straker L. "Evidence to support using squat, semi-squat and stoop techniques to lift low-lying objects". International Journal of Industrial Ergonomics 31 (2003): 149-160.

23. Selinger JC and Donelan JM. "Estimating instantaneous energetic cost during non-steady-state gait". Journal of Applied Physiology 117 (2014): 1406-1415.

24. Selinger JC., et al. "Humans can continuously optimize energetic cost during walking". Current Biology 25 (2015): 24522456.

25. Chisholm D., et al. "Physical activity readiness". British Columbia Medical Journal 17 (1975): 375-378.

26. Hermens HJ., et al. "Development of recommendations for SEMG sensors and sensor placement procedures". Journal of electromyography and Kinesiology 10 (2000): 361-374.
27. Ng J., et al. "Muscle fibre orientation of abdominal muscles and suggested surface EMG electrode positions". Electromyography and Clinical Neurophysiology 38 (1997): 51-58.

28. Surface Electromyography for the Non-Invasive Assessment of Muscles.

29. Criswell E. "Cram's introduction to surface electromyography". Jones and Bartlett Publishers (2010).

30. Marshall P and Murphy. "The validity and reliability of surface EMG to assess the neuromuscular response of the abdominal muscles to rapid limb movement". Journal of Electromyography and Kinesiology 13 (2003): 477-489.

31. Hof AL. "An explicit expression for the moment in multibody systems". Journal of Biomechanics 25 (1992): 1209-1211.

32. Plamondon A., et al. "Validation of two 3-D segment models to calculate the net reaction forces and moments at the L5S1 joint in lifting". Clinical Biomechanics 11 (1996): 101-110.

33. Burgess-Limerick R., et al. "Self-selected manual lifting technique: functional consequences of the interjoint coordination". Human Factors 37 (1995): 395-411.

\section{Volume 2 Issue 11 November 2019 (C) All rights are reserved by Mohammad} Abdoli-Eramaki. 